\title{
A NEW APPROXIMATE RIEMANN SOLVER APPLIED TO HLLC METHOD
}

\author{
Sergio Elaskar ${ }^{1,2}$, Oscar Falcinelli ${ }^{1}$ and José Tamagno ${ }^{1}$ \\ ${ }^{1}$ Departamento de Aeronáutica, FCEFyN, Universidad Nacional de Córdoba (Argentina); \\ ${ }^{2}$ Consejo Nacional de Ciencia y Tecnología, CONICET (Argentina);
}

\begin{abstract}
In solving Euler equations applying finite volume techniques, the calculations of numerical fluxes across cell interfaces, have become an essential item. The numerical scheme exactitude, the ability of handle discontinuities and the correct prediction of the propagating waves velocity, are strongly dependent on such numerical fluxes. The pioneer work of Godunov [1] was the starting point to solve the Euler equations by means of Riemann solvers. The excellent results obtained with Godunov technique, motivated several lines of research with the purpose of extending it to three dimensional flows, to achieve higher order of accuracy, etc. All calculating schemes that incorporate Riemann solvers are very precise, but unfortunately, computational demands are intense because of the non linear system of algebraic equations which must be solved in an iterative manner. An alternative which will demand less computational effort, could be provided by the use of approximate Riemann solvers, although less accurate and also, less robust. In this paper, an approximate Riemann solver which does not require iterations, possesses a high degree of accuracy and a lower computational demand in solving the Euler equations, is described. It is based on the use of dimensional analysis to reduce the number of independent variables needed to outline the physics of the problem. The scheme here presented is compared in accuracy as well as in computational effort with an exact iterative solver and with three well known approximated solvers: the Two Rarefactions Riemann Solver, the Two Shocks Riemann Solver, and an Adaptive version of these two. Substantially smaller mean errors have been found with the approximation here presented than those found with the best of all the above mentioned approximated solvers. Finally, a finite volume computer code to solve one-dimensional Euler equations using the Harten, Lax and van Leer Contact (HLLC) scheme, was developed. Results obtained solving the Shock Tube problem with the HLLC scheme, have shown no significant differences in accuracy and robustness when either the new approximate Riemann solver or the exact solver, are used. From the point of view of computer resources, the new approximate solver offers advantages.
\end{abstract}

Key words: Riemann solver, Euler equations, HLLC 


\section{INTRODUCTION}

In finite volume numerical simulations of Euler equations, the numerical fluxes evaluation at cells interfaces is a fundamental subject. Numerical fluxes have strong influence on the finite volume method accuracy, on its ability to capture discontinuities and on the correct prediction of wave velocities.

For solutions that have some degree of continuity in the functions and in their derivatives, the numerical fluxes between cells can be computed by means of truncated series expansions. However, these continuity restrictions are not verified when discontinuous solutions as shock or contact waves are present in the flow. To overcome difficulties in capturing discontinuities Godunov published his work titled "A finite difference method for the computation of discontinuous solutions of the equations of Fluid Dynamics" [1]. In this paper, an alternative approach for solving the system of Euler equations was introduced. The new Godunov approach was basically supported by physical considerations and the essential part of it is the so called Riemann solvers.

Based on the Godunov technique several more sophisticated finite volume numerical schemes for higher dimensional applications were later developed achieving second order accuracy and total variation diminishing (TVD) properties [2], [3], [4]. However, these sophisticated schemes were constructed around in the implementation of Riemann solvers, making them generally very accurate but computationally expensive because it is necessary to solve iteratively the five nonlinear algebraic equations system needed to reach an exact solution of the Riemann problem in all cells of the mesh. There are computationally less demanding techniques based on approximate solutions of the Riemann problem, but such alternative schemes have less accuracy and robustness [2].

In this paper a new non-iterative scheme, based on dimensional analysis to solve the Riemann problem applicable to time dependent and multi-dimensional Euler equations, is presented. To solve the exact Riemann problem five independent variables are involved, however using dimensional analysis, the number of variables is reduced to only three allowing the build-up of an easiest to handle data-based matrix. This new non-iterative scheme requires previously to the computation and only once, the generation of tabulated exact Riemann solutions from which the necessary information can be retrieved using linear interpolation.

\section{DESCRIPTION OF THE PROPOSED RIEMANN SOLVER}

The Euler equations govern the gas dynamic flow problems. In one dimension (1D), these equations can be written as:

$$
U_{t}+F(U)_{x}=0
$$




$$
U=\left[\begin{array}{c}
\rho \\
\rho u \\
E
\end{array}\right] ; \quad F(U)=\left[\begin{array}{c}
\rho u \\
\rho u^{2}+p \\
(E+p) u
\end{array}\right]
$$

Where $U$ is the vector of conservative variables, $F$ the vector of convective flows, $t$ and $x$ are the temporal and spatial coordinates respectively, $\rho$ is the density, $u$ the velocity in the $x$ direction, $p$ the pressure and $E$ the total energy per unit volume. The subscript means differentiation with respect to time and space.

For the Euler equations in a 1D spatial domain, the Riemann problem is defined as a particular initial value problem where the initial conditions are given by only two different constant states separated by a discontinuity. The solution of this 1D Riemann problem is selfsimilar, so that it depends directly on the ratio $x / t$, and it will consist of three types of waves: two nonlinear, shock waves or expansion fans, and one linearly degenerate, the contact discontinuity. These three waves are separating four constant states where the conservative vector $U$ acquires from the left to the right the following values $U_{L}, U_{L^{*}}, U_{R^{*}}$ and $U_{R}$. The subscripts " $L$ " and " $R$ " indicate left and right, respectively, and the symbol "*" identify points located in the state between the nonlinear waves (star region).

The evaluation of the flow variations across expansion and contact waves is realized using Riemann invariants and across shock waves, it is made by Rankine-Hugoniot relations. In gas dynamic 1D Riemann problems, this leads to an algebraic nine equations system with nine unknowns: the three waves velocities and six variables necessary to define the states $U_{L^{*}}$, $U_{R^{*}}$. However, the Euler equations eigenstructure establishes that both pressure $p_{*}$ and particle velocity $u_{*}$ are constant across the contact discontinuity, while the density take the two constant values $\rho *_{L}$ and $\rho_{*_{R}}$ [2]. Finally, using these considerations, two equations to solve the Riemann problem are implemented:

$$
\begin{gathered}
f_{L}\left(p_{*}, U_{L}\right)+f_{R}\left(p_{*}, U_{R}\right)+\Delta u=0 \\
u^{*}=\frac{1}{2}\left(u_{L}+u_{R}\right)+\frac{1}{2}\left[f_{R}\left(p_{*}, U_{R}\right)-f_{L}\left(p_{*}, U_{L}\right)\right]
\end{gathered}
$$

where $\Delta u=u_{R}-u_{L}$. The unknown for the Equations ( $3 \mathrm{a}$ and $3 \mathrm{~b}$ ) are the pressure $p *$ and the velocity $u *$. The remaining variables are calculated using the standard gas dynamic equations.

The relations across the left and right non-linear wave are represented by the functions $f_{L}$ and $f_{R}$ respectively:

$$
\begin{aligned}
& f_{K}\left(p_{*}, U_{K}\right)=\left(p_{*}-p_{K}\right) \cdot \sqrt{\frac{A_{K}}{p_{*}+B_{K}}} \quad \text { if } \quad p_{*} \geq p_{K} \\
& f_{K}\left(p_{*}, U_{K}\right)=\frac{2 \cdot a_{K}}{\gamma-1} \cdot\left[\left(\frac{p_{*}}{p_{K}}\right)^{\frac{\gamma-1}{2 \cdot \gamma}}-1\right] \quad \text { if } \quad p_{*}<p_{K}
\end{aligned}
$$




$$
A_{K}=\frac{2}{(\gamma+1) \cdot \rho_{K}} \quad B_{K}=\frac{(\gamma-1)}{(\gamma+1)} \cdot p_{K}
$$

where, $\gamma$ is the ratio of specific heats, $K$ may be $L$ or $R$ depending on if the flow changes are evaluated across the nonlinear left or right waves, and $a_{\mathrm{K}}$ is the sound velocity in the left or right states.

The Eq. (3a) is an implicit algebraic nonlinear equation on the only unknown $p_{*}$. To solve this equation an iterative scheme must be implemented. Once the Eq. (3a) is solved and the star region pressure has been obtained, it is possible to calculate using explicit expressions the velocity in the star region $u *$ and the density at each side of the contact discontinuity $\rho_{* L}$ and $\rho * R$.

Note that Eq. (3a) does not explicitly depend from $u_{L}$ or from $u_{R}$. It depends only on the difference $\Delta u=u_{R}-u_{L}$. Therefore the pressure in the star zone becomes only function of five variables:

$$
p_{*}=f_{1}\left(\Delta u, \rho_{L}, p_{L}, \rho_{R}, p_{R}\right)
$$

To reduce the number of independent variables necessaries to describe the gasdynamic Riemann problem, the dimensional analysis is used. Consider as reference variables the density and pressure from the Riemann problem right side

$$
\rho_{\text {ref }}=\rho_{R} \quad p_{\text {ref }}=p_{R}
$$

the densities can then be written in non-dimensional form using a reference value $\rho_{R}$, the pressures using as reference $p_{R}$, and the velocities using $\left(\rho_{R} p_{R}\right)^{1 / 2}$. Therefore, the solution of a particular Riemann problem is governed by only three parameters:

$$
\pi_{1}=\frac{\Delta u}{\sqrt{\frac{p_{R}}{\rho_{R}}}} \quad \pi_{2}=\frac{p_{L}}{p_{R}} \quad \pi_{3}=\frac{\rho_{L}}{\rho_{R}}
$$

In Table 1 are indicated seven different cases of Riemann problems with identical values of $\pi_{1}, \pi_{2}, \pi_{3}$, satisfying the parameters $\pi_{1}=-0.78262, \pi_{2}=50, \pi_{3}=10$. The relation $p_{*} / p_{R}$ is obtained solving Eqs. ( 3 and 4 ), and for the seven test cases considered it is obtained $p * / p_{R}=$ 13.312 , which shows that the proposed non-dimensional analysis works correctly. Then, it is possible to write the Eq. (3) only as function of $\pi_{1}, \pi_{2}, \pi_{3}$ and $p * / p_{R}$. 
Table 1. Test cases.

\begin{tabular}{|c|c|c|c|c|c|}
\hline$\rho_{L}$ & $u_{\mathrm{L}}$ & $p_{\mathrm{L}}$ & $\rho_{R}$ & $u_{\mathrm{R}}$ & $p_{R}$ \\
\hline $1.2250 \mathrm{E}+00$ & $1.0000 \mathrm{E}+02$ & $1.0000 \mathrm{E}+05$ & $1.2250 \mathrm{E}-01$ & $0.0000 \mathrm{E}+00$ & $2.0000 \mathrm{E}+03$ \\
\hline $4.9071 \mathrm{E}+01$ & $8.4770 \mathrm{E}+02$ & $8.7460 \mathrm{E}+06$ & $4.9071 \mathrm{E}+00$ & $6.9994 \mathrm{E}+02$ & $1.7492 \mathrm{E}+05$ \\
\hline $6.7304 \mathrm{E}+00$ & $6.5231 \mathrm{E}+02$ & $1.0554 \mathrm{E}+07$ & $6.7304 \mathrm{E}-01$ & $2.1402 \mathrm{E}+02$ & $2.1108 \mathrm{E}+05$ \\
\hline $4.1503 \mathrm{E}+00$ & $7.8027 \mathrm{E}+02$ & $1.1631 \mathrm{E}+07$ & $4.1503 \mathrm{E}-01$ & $1.9437 \mathrm{E}+02$ & $2.3261 \mathrm{E}+05$ \\
\hline $9.4504 \mathrm{E}+00$ & $6.4262 \mathrm{E}+02$ & $1.5976 \mathrm{E}+07$ & $9.4504 \mathrm{E}-01$ & $1.8756 \mathrm{E}+02$ & $3.1952 \mathrm{E}+05$ \\
\hline $3.0289 \mathrm{E}+01$ & $2.9038 \mathrm{E}+02$ & $2.9757 \mathrm{E}+06$ & $3.0289 \mathrm{E}+00$ & $1.8067 \mathrm{E}+02$ & $5.9514 \mathrm{E}+04$ \\
\hline $3.6284 \mathrm{E}+01$ & $3.0129 \mathrm{E}+02$ & $6.5687 \mathrm{E}+05$ & $3.6284 \mathrm{E}+00$ & $2.5420 \mathrm{E}+02$ & $1.3137 \mathrm{E}+04$ \\
\hline
\end{tabular}

Since in solving the Eq. (3) there are involved only three independent variables, a data-base matrix with three degrees of freedom containing the values of $p_{*} / p_{R}$ for $\mathrm{N}$ values of $\pi_{1}$, M of $\pi_{2}$, and $\mathrm{Q}$ of $\pi_{3}$ is, in situ generated. Then, to find the solution of a particular Riemann problem, it is only necessary to calculate the corresponding values of $\pi_{1}, \pi_{2}, \pi_{3}$ and to interpolate for $p * / p_{R}$ in the $\mathrm{N} \times \mathrm{M} \times \mathrm{Q}$ matrix (from now, simply called A-matrix). Finally the pressure in the star zone can be calculated as:

$$
p_{*}=\overline{\left(\frac{p_{*}}{p_{R}}\right)} \cdot p_{R}
$$

where $\left(\overline{p_{*} / p_{R}}\right)$ is the interpolated value from the A-matrix. After calculating the pressure in the star region, the rest of the variables can be explicitly calculated using the same equations of the exact solver [2].

The previously described procedure involves the use of an interpolated value of pressure to calculate the density and velocity changes across each wave. However, it is not the only possible procedure. Others alternatives are to develop arrangements with dimensionless density or dimensionless velocity variations across each wave.

To avoid unneeded data storage and to increase the approximate solution accuracy, the variation range of the parameters $\pi_{1}, \pi_{2}, \pi_{3}$ must be as small as possible. To do this, symmetrical Riemann problems are considered. Two Riemann problems are symmetric if the following conditions are verified:

$$
\left(p_{R}\right)_{A}=\left(p_{L}\right)_{B} \quad\left(\rho_{R}\right)_{A}=\left(\rho_{L}\right)_{B} \quad \Delta u_{A}=\Delta u_{B}
$$

Also, for symmetrical Riemann problems the unnecessary storage can be avoided if as reference variables are adopted not the left or right pressure and density but instead, those of the higher pressure side. Thus, the reference state is the higher initial state pressure and the $\pi_{2}$ value will always be less than or at most equal, to one.

To give a physical sense to the non-dimensional $\pi_{1}$ variable it is convenient to re-define it as: 


$$
\pi_{1}=\frac{\Delta u}{\sqrt{\gamma \cdot \frac{p_{r e f}}{\rho_{r e f}}}}=\frac{\Delta u}{a_{r e f}}
$$

$a_{\text {ref }}$ is the sound velocity in the reference state and $\pi_{1}$ would be like a Mach number change between the left and right states in the Riemann problem. Note that $\pi_{1}$ does not represent strictly a Mach number change because $a_{\text {ref }}$ is only the sound velocity in the left or in the right state.

When the Riemann problem solution has left and right expansion waves it is not necessary to any interpolation scheme, because the Eq. (3) has a close form solution.

\section{COMPARISON WITH OTHER RIEMANN SOLVERS}

To study the computational efficiency and accuracy of the proposed Riemann solver, comparisons with others Riemann solvers [2] are carried out: one finds iteratively the exact solution of Eq. (3a), and others two that use approximate schemes. To make the comparison, Riemann problems were generated randomly with values of the parameters $\pi_{1}, \pi_{2}$ and $\pi_{3}$ ranging between:

$$
-10.05 \leq \pi_{1} \leq 4.95 \quad 0.05 \leq \pi_{2} \leq 1 \quad 0.05 \leq \pi_{3} \leq 5.05
$$

\subsection{Iterative Riemann Solver}

This solver searches iteratively a solution of the Riemann problem. The Eq.(3a) for any value of $p$ can be written as:

$$
f_{L}\left(p, U_{L}\right)+f_{R}\left(p, U_{R}\right)+\Delta u=R(p)
$$

where $R(p)$ is the residual to cancel; $f_{L}$ and $f_{R}$, are calculated according to Equations (4 and 5).

To implement the Newton-Raphson method to solve the Eq. (13) calculations of the function and of its derivative are necessary which, undoubtedly should increase the computational cost. However, this increase is not significant because the evaluation of the derivative demands simple computations once the function has been evaluated. As $U_{R}$ and $U_{L}$ are constant the derivative of the residual function, Eq. (13), is calculated using only the functions $f_{L}$ and $f_{R}$. The derivative can then be written as:

$$
\frac{d\left[f_{L}\left(p, U_{L}\right)\right]}{d p}+\frac{d\left[f_{R}\left(p, U_{R}\right)\right]}{d p}=\frac{d[R(p)]}{d p}
$$

Where, the derivatives $\frac{d\left[f_{L}\left(p, U_{L}\right)\right]}{d p}=f_{L}^{\prime}$ and $\frac{d\left[f_{R}\left(p, U_{R}\right)\right]}{d p}=f_{R}^{\prime}$ are: 


$$
f_{K}^{\prime}=\left\{\begin{array}{c}
\sqrt{\frac{A_{K}}{B_{K}+p} \cdot\left(1-\frac{p-p_{K}}{2 \cdot\left(B_{K}+p\right)}\right) \text { if } p \geq p_{K}} \\
\frac{1}{\rho_{\mathrm{K}} \cdot a_{\mathrm{K}}} \cdot\left(\frac{p}{p_{\mathrm{K}}}\right)^{\frac{-(\gamma+1)}{2 \cdot \gamma}} \text { if } p<p_{K}
\end{array}\right.
$$

It is noted that, from the computational point of view, the most expensive steps involved in the numerical evaluation of Eq.(15) are the powers with fractional exponents.

The Newton-Raphson algorithm applied to Eq.(13) can be written as:

$$
p_{i+1}=p_{i}-\frac{R_{\left(p_{i}\right)}}{R_{\left(p_{i}\right)}^{\prime}}
$$

$p_{i}$ and $p_{i+1}$ are the pressure for the iteration $i$ and $i+1$ respectively, $R$ and $R$ are the residual function and its derivative.

During the iterative solution of the Eq. (13) difficulties may appears because the pressure can becomes negative (see Eq. (16)). To avoid this disadvantage, the residual function is evaluated considering $p=p_{\min }$ and $p=p_{\max }$, where $p_{\min }=\min \left[p_{L} p_{R}\right]$ and $p_{\max }=$ $\max \left[p_{L} p_{R}\right]$. If both residuals are positive, the pressure which cancels the residual is less than $p_{L}$ and $p_{R}$, and the Riemann problem has an explicit solution consisting of two rarefaction waves. If the residual corresponding to the maximum pressure is greater than zero and the corresponding to minimum pressure is less than zero, the Riemann problem have as solutions a shock wave and a rarefaction fan, and it is adopted as a first iteration the value of the minimum pressure (which will undoubtedly be lower than the pressure to cancel the residual). When both residuals are negative, the Riemann problem has two shock waves and the sought pressure will be greater than both, so as first iteration the maximum pressure value is adopted.

\subsection{Two-Rarefaction Riemann Solver (TRRS)}

A particular solution of the gas-dynamics Riemann problem is produced when both non-linear waves are rarefaction waves. In this case the pressure in the star region can be obtained analytically: 


$$
\begin{gathered}
\frac{2 \cdot a_{L}}{\gamma-1} \cdot\left[\left(\frac{p_{*}}{p_{L}}\right)^{\frac{\gamma-1}{2 \cdot \gamma}}-1\right]+\frac{2 \cdot a_{R}}{\gamma-1} \cdot\left[\left(\frac{p_{*}}{p_{R}}\right)^{\frac{\gamma-1}{2 \cdot \gamma}}-1\right]+\Delta u=0 \\
\Downarrow \\
p_{*}=\left[\frac{a_{L}+a_{R}-\Delta u \cdot \frac{(\gamma-1)}{2}}{\left.\frac{a_{L}}{p_{L}^{\left(\frac{\gamma-1}{2 \cdot \gamma}\right)}+\frac{a_{R}}{p_{R}^{\left(\frac{\gamma-1}{2 \cdot \gamma}\right)}}}\right]^{\left(\frac{2 \cdot \gamma}{\gamma-1}\right)}}\right.
\end{gathered}
$$

The TRRS method is generally robust and it calculates the pressure in the star region always using the Eq.(17), no matter what kind of Riemann problem is studied. The error of this method will be null for the Riemann problems with two rarefaction waves $\left(p_{*} \leq p_{\min }\right)$ and will increase as $p_{*}$ becoming higher and moves away from $p_{\text {min. }}$.

\subsection{Two-Shock Riemann solver (TSRS)}

The TSRS searches the solution of the Riemann problem assuming that both nonlinear waves are shocks. Then the functions $f_{K}$ are given by the Eq.(4b) and the Eq.(3a) can be written as:

$$
\begin{gathered}
\left(p_{*}-p_{L}\right) \cdot \sqrt{\frac{2}{\frac{(\gamma+1) \cdot \rho_{L}}{p_{*}+\frac{(\gamma-1)}{(\gamma+1)} \cdot p_{L}}}+\left(p_{*}-p_{R}\right) \cdot \sqrt{\frac{\frac{2}{(\gamma+1) \cdot \rho_{R}}}{p_{*}+\frac{(\gamma-1)}{(\gamma+1)} \cdot p_{R}}}+\Delta u=0} \\
\Downarrow \\
\left(p_{*}-p_{L}\right) \cdot g\left(p_{*}, U_{L}\right)+\left(p_{*}-p_{R}\right) \cdot g\left(p_{*}, U_{R}\right)+\Delta u=0
\end{gathered}
$$

where

$$
g\left(p, U_{K}\right)=\sqrt{\frac{\frac{2}{(\gamma+1) \cdot \rho_{K}}}{p+\frac{(\gamma-1)}{(\gamma+1)} \cdot p_{K}}}
$$

The TSRS does not provide an analytical solution for $p_{*}$, therefore it is necessary to implement an iterative process beginning with an initial approximation for $p_{*}$ called $p_{0} . p_{0}$ is utilized to evaluate the initial values of $g\left(p_{0}, U_{L}\right)$ and $g\left(p_{0}, U_{R}\right)$. Then, assuming $g\left(p_{0}, U_{L}\right)$ and $g\left(p_{0}, U_{R}\right)$ constants the Eq.(18) is lineal and it is possible to obtain $p_{*}$ as: 


$$
p_{*}=\frac{p_{L} \cdot g\left(p_{0}, U_{L}\right)+p_{R} \cdot g\left(p_{0}, U_{R}\right)-\Delta u}{g\left(p_{0}, U_{L}\right)+g\left(p_{0}, U_{R}\right)}
$$

The $p_{0}$ value can be expressed as [2]:

$$
p_{0}=\frac{p_{L}+p_{R}}{2}+\frac{u_{L}+u_{R}}{2} \cdot \frac{\rho_{L}+\rho_{R}}{2} \cdot \frac{a_{L}+a_{R}}{2}
$$

The TSRS scheme, even when applied to cases with two shock waves cannot provide an exact solution; it is very.

\subsection{Approximate and Adaptive Riemann solver using the TSRS and TRRS}

This approximate Riemann solver binds together the advantages of the TRRS and TSRS schemes. This adaptive Riemann solver calculates the approximate $p_{0}$ as given by Eq.(21), then compare this pressure with $p_{\min }$ and $p_{\max }$, and the pressure in the star region will be:

$$
p_{*}= \begin{cases}p_{* T R R S} & \text { if } p_{0} \leq p_{\text {min }} \\ p_{0} & \text { if } p_{\text {min }}<p_{0}<p_{\text {máx }} \\ p_{* T S R S} & \text { if } p_{0} \geq p_{\text {máx }}\end{cases}
$$

being $p_{* T R R S}$ and $p_{* T S R S}$ the pressures obtained using the TRRS and TSRS solvers respectively.

\subsection{Results using approximate Riemann solvers}

To compare the behavior of the proposed Riemann solver with the others Riemann solvers, $10^{6}$ Riemann problems were evaluated using values of the parameters $\pi_{i}$ within the ranges given by Eq. (12). Of them, 65\% were cases with two shock waves, $6 \%$ with one shock and one rarefaction wave and the remaining $29 \%$ with two rarefaction waves.

To systematized the study, in all Riemann problems the following initial conditions to the right state are established

$$
\rho_{R}=1 \frac{k g}{m^{3}} \quad u_{R}=0 \frac{m}{s} \quad p_{R}=1 \frac{N}{m^{2}}
$$

The initial conditions for the left states are calculated using values of the parameters $\pi_{l}$, $\pi_{2}$ and $\pi_{3}$ :

$$
u_{L}=-\pi_{1} \sqrt{r \frac{p_{R}}{\rho_{R}}} \quad p_{L}=\pi_{2} p_{R} \quad \rho_{L}=\pi_{3} \rho_{R}
$$


To compare the approximate Riemann solvers behaviors, in the following table are listed the average error of all the pressures computed in the star region for the approximate solvers. The results are given in $\mathrm{N} / \mathrm{m}^{2}$ :

Table 2. Average error.

\begin{tabular}{|c|c|c|c|}
\hline TRRS & TSRS & Adaptive & New scheme \\
\hline 37.371 & 7.1504 & 7.0931 & 0.0019 \\
\hline
\end{tabular}

Another variable used to compare the Riemann solvers behavior is the worst approximate test values for the TRRS, the TSRS, the adaptive RS and the new proposed scheme. The results are presented in Table 3:

Table 3. Worst approximated solutions after solving Riemann test cases.

\begin{tabular}{|c|c|c|c|c|c|c|c|c|}
\hline Solver & $\rho_{L}$ & $u_{L}$ & $p_{L}$ & $\rho_{R}$ & $u_{R}$ & $p_{R}$ & $\begin{array}{c}\text { Solver } \\
\text { prediction }\end{array}$ & $\begin{array}{c}\text { Exact } \\
\text { solution }\end{array}$ \\
\hline TRRS & 4.9733 & 11.8082 & 0.0507 & 1 & 0 & 1 & 998.7362 & 81.2775 \\
\hline TSRS & 4.9182 & 11.8582 & 0.0564 & 1 & 0 & 1 & 31.8961 & 81.6784 \\
\hline Adaptive & 4.9182 & 11.8582 & 0.0564 & 1 & 0 & 1 & 31.8961 & 81.6784 \\
\hline Proposed & 0.0739 & 11.8752 & 0.9274 & 1 & 0 & 1 & 9.5344 & 9.6541 \\
\hline
\end{tabular}

Note that for the three first solvers, the worst results appear when there are relatively strong shock waves. Also, it is noted that the most poorly test case predicted is the same for the TSRS and the Adaptive Riemann solvers. This is so because when the star pressure is highest that both initial pressures, the Adaptive solver uses the same calculation scheme that the TSRS.

The percent error for each tested Riemann solvers is (see Table 4):

Table 4. Percent error for Riemann solvers.

\begin{tabular}{|c|c|c|c|}
\hline TRRS & TSRS & Adaptive & New scheme \\
\hline $1128.80 \%$ & $60.95 \%$ & $60.95 \%$ & $1.24 \%$ \\
\hline
\end{tabular}

In Table 5 the necessary CPU time by each approximate solver is given as a percentage of the necessary CPU time for the exact Riemann solver:

Table 5. Percent of CPU time.

\begin{tabular}{|c|c|c|c|}
\hline TRRS & TSRS & Adaptive & New scheme \\
\hline $38.62 \%$ & $25.93 \%$ & $31.60 \%$ & $34.57 \%$ \\
\hline
\end{tabular}

To avoid the formation of high-intensity shocks and near-vacuum conditions, the restriction that the exact pressure $p^{*}$ in each case is bounded to $0.1 p_{L}<p^{*}<10 p_{L}$ and $0.1 p_{R}$ 
$<p^{*}<10 p_{R}$, it is added, and the previous analysis are repeated. The worst computed cases for each solver are shown in Table 6:

Table 6. Worst approximated solutions after solving Riemann test cases with restrictions in $p^{*}$.

\begin{tabular}{|c|c|c|c|c|c|c|c|c|}
\hline Solver & $\rho_{L}$ & $u_{L}$ & $p_{L}$ & $\rho_{R}$ & $u_{R}$ & $p_{R}$ & $\begin{array}{c}\text { Solver } \\
\text { prediction }\end{array}$ & $\begin{array}{c}\text { Exact } \\
\text { solution }\end{array}$ \\
\hline TRRS & 2.4112 & 4.2348 & 0.9999 & 1 & 0 & 1 & 12.5554 & 9.9950 \\
\hline TSRS & 0.8759 & 5.3169 & 1.0013 & 1 & 0 & 1 & 6.7847 & 9.9618 \\
\hline Adaptive & 0.8759 & 5.3169 & 1.0013 & 1 & 0 & 1 & 6.7847 & 9.9618 \\
\hline Proposed & 0.0712 & 11.7759 & 0.9424 & 1 & 0 & 1 & 9.2260 & 9.3234 \\
\hline
\end{tabular}

The percent error for each of the tested Riemann solvers using a bounded $p^{*}$ are:

Table 7. Percent errors.

\begin{tabular}{|c|c|c|c|}
\hline TRRS & TSRS & Adaptive & New scheme \\
\hline $25.62 \%$ & $31.89 \%$ & $31.89 \%$ & $1.04 \%$ \\
\hline
\end{tabular}

From all the previous results, it is concluded that the new approximate Riemann solver works very well and reduces signficantly the errors introduced by others approximated solvers.

\section{DESCRIPTION OF THE ONE-DIMENSIONAL HLLC TVD METHOD}

To test the usefulness of the proposed Riemann solver, a computer code was developed using the one-dimensional versions of the HLLC (Harten, Lax, van Leer Contact) method [2], [5].

In Figure 1, it is shown the wave structure utilized by the HLLC method. It contains the slowest $S_{L}$ and fastest $S_{R}$ waves and a middle one of speed $S_{*}$. Note that the HLLC solver does not calculate the $S_{L}$ and $S_{R}$ wave velocities; which are estimated using a Riemann solver. For the purpose of comparison, in this paper are implemented the new approximate Riemann solver and the exact Riemann solver. 


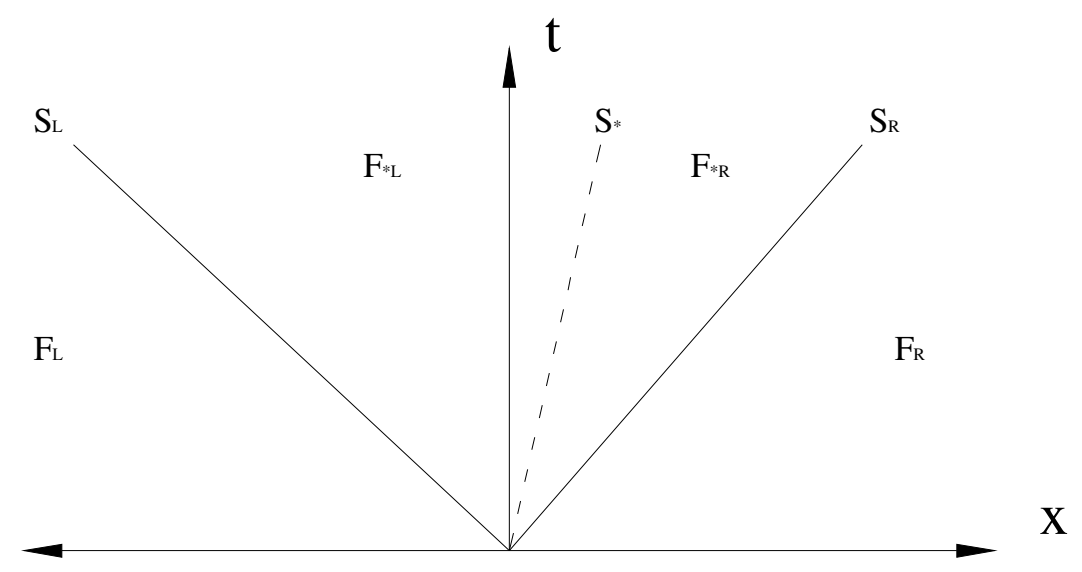

Figure 1. Riemann problem for the HLLC method.

By applying Rankine-Hugoniot conditions across each of the three waves $S_{L}, S_{*}, S_{R}$ it can be obtained

$$
\begin{gathered}
\text { R.H. } \Rightarrow F_{*_{L}}-F_{L}=S_{L} \cdot\left(U_{*_{L}}-U_{L}\right) \\
\text { R.H. } \Rightarrow F_{*_{R}}-F_{*_{L}}=S_{*} \cdot\left(U_{*_{R}}-U_{*_{L}}\right) \\
\text { R.H. } \Rightarrow F_{R}-F_{*_{R}}=S_{R} \cdot\left(U_{R}-U_{*_{R}}\right)
\end{gathered}
$$

These are three equations for the four unknowns vectors $U_{*_{L} L} U_{*_{R}}, F_{*_{L}}$ and $F_{*_{R}}$. Using the Eqs. $(25)$ the fluxes $F_{*_{L}}$ and $F_{*_{R}}$ can be determined as:

$$
U_{* R} \cdot\left(S_{R}-S_{*}\right)+U_{* L} \cdot\left(S_{*}-S_{L}\right)=F_{L}-F_{R}+S_{R} \cdot U_{R}-S_{L} \cdot U_{L}
$$

Eq. (26) is formed by a combination of three scalar equations and seven unknowns, the components of $U_{*_{L}}$ and $U_{*_{R}}$ states and $\mathrm{S}_{*}$. This implies that $S_{L}$ and $S_{R}$ must be known, which can be evaluated by a Riemann solver. However, the exact solution satisfies the following conditions:

$$
u_{*_{R}}=u_{*_{L}}=u_{*} \quad p_{*_{R}}=p_{*_{L}}=p_{*}
$$

and moreover, the star zone velocity is equal to the contact discontinuity velocity. That is:

$$
S_{*}=u *
$$

The last closure condition is given by [2]:

$$
\rho_{*_{K}}=\rho_{K} \cdot\left(\frac{S_{K}-u_{K}}{S_{K}-S_{*}}\right)
$$


To avoid confusion the first, second and third scalar components of the RHS vector of Eq. (26), are called now RHS1, RHS2 and RHS3 respectively. It can readily be show that Eqs. (26 and 27) are simultaneously satisfied by the relations:

$$
\begin{gathered}
S_{*}=\frac{R H S 2}{R H S 1} \\
p_{*}=\frac{R H S 3-\frac{S_{*}}{2} \cdot R H S 2}{\left(S_{R}-S_{L}\right)}(r-1)
\end{gathered}
$$

Notice that to obtain Eq. (30) used is made of the relation $E=\frac{\rho}{2} u^{2}+\frac{p}{\gamma-1}$.

The complete calculation scheme shall consist in first obtaining an estimation of $S_{L}$ and $S_{R}$, needed in the calculations of $S_{*}$ and $p_{*}$ using Eqs (29 and 30), respectively. Then, determining $\rho *_{R}$ and $\rho *_{L}$ through Eq. (28) and finally the fluxes $F_{*_{L}}$ and $F_{*_{R}}$ using Eqs. (25).

Calling wave-1, wave-2 and wave-3 those that separate the $L$ and $* L, * L$ and $* R$, $* R$ and $R$ states respectively; the algorithm in this paper implemented to compute the numerical fluxes is

$$
F_{i+1 / 2}=\frac{1}{2}\left(F_{i}+F_{i+1}\right)-\frac{1}{2} \sum_{j=1}^{3} \operatorname{sign}\left(S_{j}\right) \phi_{i+1 / 2}^{j} \Delta F_{i+1 / 2}^{j}
$$

being $i$ and $i+1$ the left and right cells and $\Delta F_{i+1 / 2}^{j}=F_{i+1 / 2}^{j+1}-F_{i, 1 n}^{j}$ : the fluxes at the cells interfaces $F_{i+1 / 2}^{j}$ are calculated by means of the HLLC scheme. $\phi_{i+1 / 2}^{j}$ is the limiter function for the wave-j. In this work only the results obtained using a Van Leer limiter function [6] are presented, however the scheme works efficiently with other limiters. Finally, the flow state vector is actualized at each time step by means of the explicit time advance scheme:

$$
U_{i}^{n+1}=U_{i}^{n+1}+\frac{\Delta t}{\Delta x}\left(F_{i-1 / 2}-F_{i+1 / 2}\right)
$$

\subsection{Results obtained using the HLLC scheme}

Two compare the performance of the HLLC method implementing the new approximate Riemann solver and the exact one, a two meters length shock tube is tested. The shock tube problem was selected because it is possible to obtain an exact solution, and is a very popular benchmark for computational gas dynamics.

The shock tube has two sections filled with air at the same temperature and of equal length separated by a diaphragm (discontinuity on the initial condition). Initially, the velocities along the tube are null. Inside the right section, the initials pressure and density are $p=10^{5} \mathrm{~N} / \mathrm{m}^{2}$ and $\rho=1.225 \mathrm{~kg} / \mathrm{m}^{3}$ respectively, and in the left section are $p=10^{4} \mathrm{~N} / \mathrm{m}^{2}$ and $\rho=0.1225 \mathrm{~kg} / \mathrm{m}^{3}$. For these initial conditions, the solution after the diaphragm is broken is is composed by a shock wave traveling to the right at $543.4 \mathrm{~m} / \mathrm{s}$, and one contact surface also 
moving to the right at $277.6 \mathrm{~m} / \mathrm{s}$. There is also, a rarefaction fan traveling to the left, its wave tail is moving at $338.1 \mathrm{~m} / \mathrm{s}$ and its front at $4.9 \mathrm{~m} / \mathrm{s}$.

The flow properties at the four states limited by the above described wave are:

Table 8. Shock tube states flow properties.

\begin{tabular}{|c|c|c|c|c|}
\hline & $L$ & $* L$ & $* R$ & $R$ \\
\hline$\rho$ & 1.225 & 0.4995 & 0.2504 & 0.1225 \\
\hline$U$ & 0 & 277.6 & 277.6 & 0 \\
\hline$P$ & 100000 & 28482 & 28482 & 10000 \\
\hline
\end{tabular}

In all tests the mesh has 200 cells evenly distributed along the tube and the results are presented in Figure 2. In this figure are shown three lines; one represents the exact solution (blue continue line), another solution is obtained using the exact Riemann solver (red with squares), and finally the third line corresponds to the results calculated using the approximated Riemann solver proposed in this work (black with triangles).

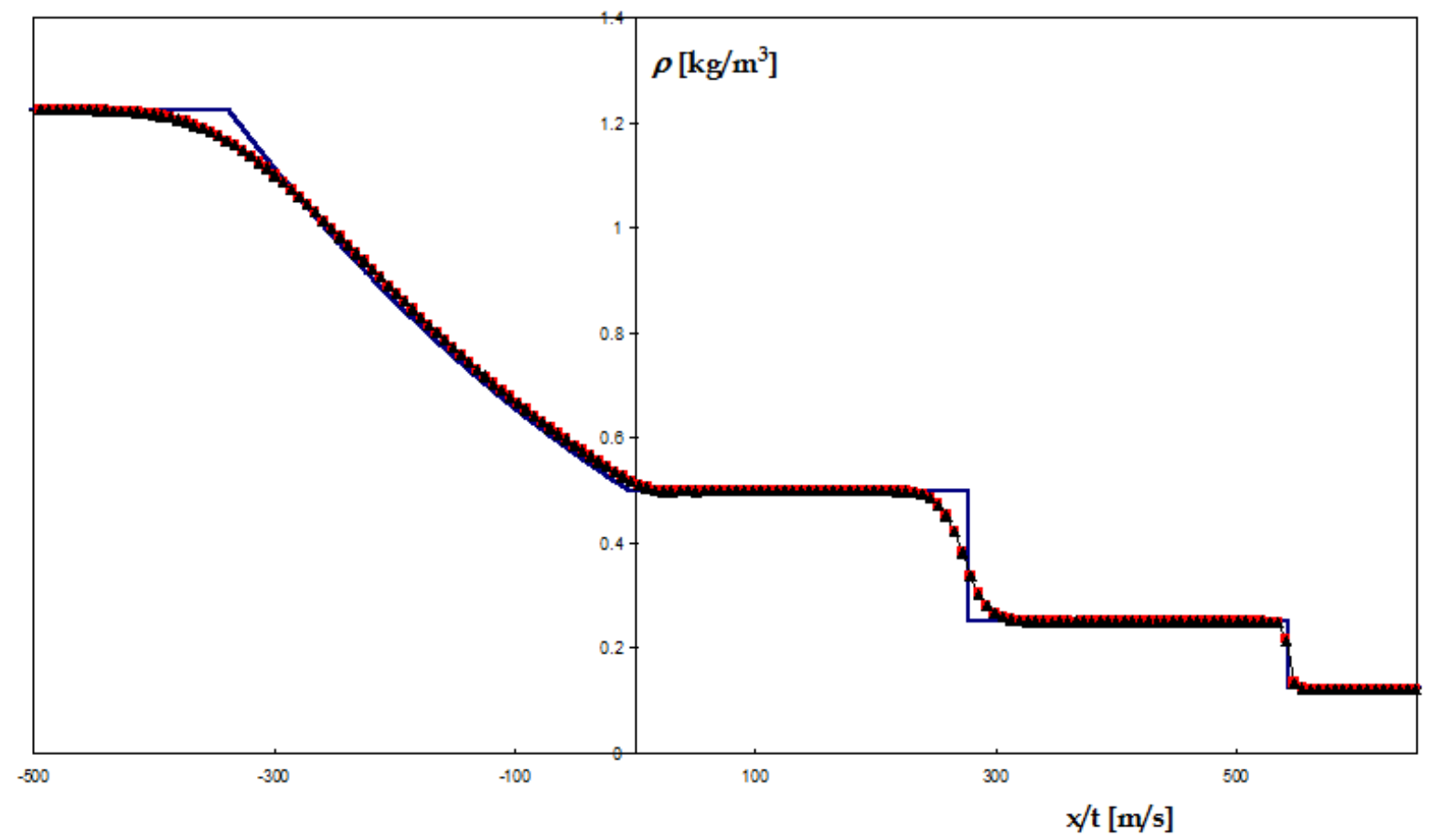

Figure 2. One-dimensional HLLC method. Blue continue line: theoretical solution. Red squares: exact solvers. Black triangles: approximate solver.

The differences between the numerical results presented in Figure 2 are less than $1 \%$. The results calculated using, either the exact Riemann solver or the new proposed approximation, are practically identical. However, the new Riemann solver reduces $20 \%$ the CPU time, approximately. 


\section{CONCLUSIONS}

From the comparison of the proposed approximated Riemann solver with the TRRS, the TSRS and the Adaptive RS, it has been found that for all star region pressure values computed, the average error of the new solver is notably smaller than the average error of all the others approximated Riemann solvers. Also, considering the worst star region pressure values, the new solver percentage error is almost 50 times smaller than the best percentage obtained by the other solvers. From the point of view of computational cost, it is higher if compared with the Adaptive RS and TSRS by $33.3 \%$ and $9.4 \%$ respectively, but lower by $11.75 \%$ in comparison with the TRRS. However, the new solver presents a higher cost-benefit ratio.

Although the proposed solver has already shown to be efficient, it still requires a sequence of operations that others solvers do not need. For instance, the matrix A must be generated (a 100x100x100 matrix like the one used in this paper, needs the exact solution of $10^{6}$ Riemann problems). However, this matrix is calculated only once at the beginning.

The numerical results obtained with HLLC scheme, have shown that the new solver is accurate, and no significant differences were found when results are compared with the exact Riemann solver. In addition, it shows advantages because reduces CPU time.

\section{Acknowledgements}

The authors would like to acknowledge and thank the support of CONICET, the SECyT of the Universidad Nacional de Córdoba, and MCyT of Provincia de Córdoba.

\section{REFERENCES}

[1] Godunov, S. (1959). A Finite Difference Method for the computation of Discontinuous Solutions of the Equations of Fluid Dynamics. Mat. Sb., Vol. 47, pp. 357-393 (In Russian).

[2] Toro, E. (2009). Riemann Solvers and Numerical Methods for Fluid Mechanics. A practical introduction (Third Edition), Springer-Verlag, ISBN 978-3-540-25202-3, Berlin.

[3] LeVeque, R. (2004). Finite Volume Methods for Hyperbolic Problems (Second Edition), Cambridge University Press, ISBN 0-521-00924-3, Cambridge.

[4] Yee, H. (1989) A Class of High Resolution Explicit and Implicit Shock-Capturing Methods. NASA Technical memorandum 101088. Ames Research Center, California.

[5] Toro, E., Spruse, M. and Speares, W. (1994). Restoration of the Contact Surface in the HLL-Riemann solver. Shock Waves, Vol. 4, pp. 25-34, ISSN 0938-1287. 
[6] van Leer, B. (1985). On the Relation Between the Upwind-Differencing Schemes of Godunov, Enguist-Osher and Roe. SIAM Journal of Scientific Computing, Vol. 5, No. 1, pp. 1-20, ISSN 1064-8275. 